\title{
Leprosy control in Zimbabwe: from a vertical to a horizontal programme
}

\author{
D K WARNDORFF \& J A WARNDORFF \\ Postbus 935, 6200 AX Maastricht, The Netherlands
}

\section{Accepted for publication 14 November 1989}

Summary In Zimbabwe leprosy control services were re-established in 1983, following the war of independence. Its main objectives were the nation-wide implementation of multiple drug treatment (MDT) and the integration of leprosy control into the general health services.

The MDT regimens have led to a rapid reduction of the prevalence of leprosy. At the beginning of 1989357 patients were on treatment and 1299 under followup. Six hundred and twenty-seven new cases have been detected since 1984, which represents an annual case detection rate of 1.6 per 100,000 . This seems a fair reflection of the incidence rate, as the new cases are characterized by a minority of patients under the age of $15(4 \%)$ and a lepromatous percentage of $50 \%$.

As the budget of the programme has remained unchanged integration of leprosy control into the general health services has become imperative. However, this transition is now hindered by a number of obstacles that were not foreseen at the start of the programme, because they are in measure corollaries of the successful implementation of MDT.

Most of the problems that leprosy control is facing in Zimbabwe could have been avoided if instruction in leprosy had been introduced into the curricula of the (para) medical training schools 20 years ago.

\section{Introduction}

In Zimbabwe a very successful leprosy control programme based on MDT was established in 1983. It led to a rapid decrease in the prevalence of leprosy; however, the cost-effectiveness of the programme declined as well. Priority for leprosy control in Zimbabwe at present is therefore its full integration into the general health services. This paper discusses the problems attending that process.

\section{Geography}

Zimbabwe lies in southern Africa, bordered by Zambia, Mozambique, Botswana and South Africa. The country has an area of nearly 400,000 sq. $\mathrm{km}$ and is divided into eight 
provinces and 55 districts. It has a good infrastructure with regard to roads, public transport and telephone communication. The capital city is Harare.

The population of Zimbabwe is 8.7 million, with an annual growth rate of $2.8 \%$. The population density varies from 10 to 60 per sq. $\mathrm{km}$. Twenty per cent of the population live in the commercial farming areas, which comprises $42 \%$ of the country.

Zimbabwe became an independent republic in 1980 .

\section{History of leprosy control in Zimbabwe}

Following the Leprosy Suppression Act of 1911, leprosy patients were 'arrested' and segregated from the community in two large leprosaria, where dapsone treatment was introduced in 1955. All patients, irrespective of their classification, were treated for life with dosages as low as $12 \cdot 5$ to $25 \mathrm{mg}$ daily.

In 1964 the leprosaria were closed and mobile leprosy control services were started. These were well organized, with a defaulter rate of less than $25 \%$. The treatment policy remained the same as in the leprosaria. Approximately 6000 patients were on register by the mid-seventies, while the prevalence was estimated at 10,000 cases ( 1.25 per thousand). The majority of the patients were living in the least developed parts of the country in the Zambezi valley, along the borders with Zambia and Mozambique.

During the war of liberation preceding Independence, leprosy control-like most of the medical services - collapsed as health centres were destroyed and rural health workers moved into the towns.

The leprosy control services were re-established in 1983 with the help of the Associzione Italiana 'Amici di Raoul Follereau'. It was to be based first, on nation-wide implementation of the WHO-recommended MDT regimens, and second, on the integration of leprosy control into the general health services. Since 1984 the latter are being organized according to primary health care principles.

As there was little leprosy-trained staff among the general health personnel, eight general health workers were given training in leprosy, both locally and at ALERT, and subsequently appointed as Provincial Leprosy Officers (PLOs). They were charged with the implementation of MDT in the provinces, and with the in-service training of general health staff. Additionally, a total of 24 leprosy 'scouts' were employed in areas of high endemicity for the detection of new cases and the follow-up of defaulters.

Table 1 summarizes the intake of patients since the start of the programme in 1984.

Table 1. It is shown that the intake of patients was greatest during the first two years when no distinction was made between previously treated patients and new cases. After 1986 the former were accepted for an additional course of MDT only if they showed clinical or bacteriological signs of activity of leprosy.

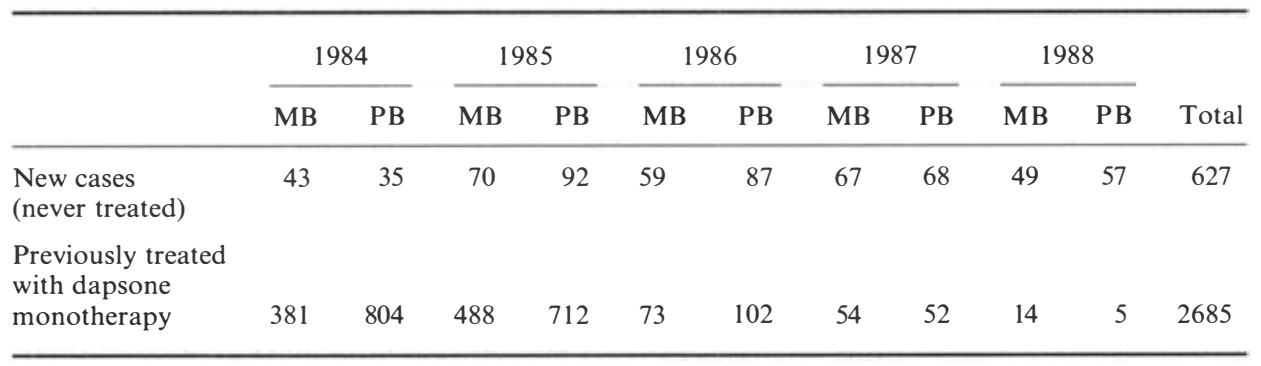


Many of the 6000 patients who were on register in the seventies were found to have died, their disease had arrested, or they had returned to their home country Malawi or Zambia.

At present the majority of the patients have completed their six months or two years of treatment, and the paucibacillary patients among them also their two years of follow-up. At the beginning of 1989,357 patients were still on treatment while 1299-mostly multibacillary cases-had not yet completed their five years of follow-up. The 627 new cases found since 1984 represent an average case detection rate of 1.6 per 100,000 inhabitants per year; this seems a fair reflection of the incidence, as the new cases are characterized by a minority of children below the age of 15 years $(4 \%)$ and by a lepromatous fraction of $50 \%$. Although the latter percentage includes all smear positive and $\mathrm{BB}$ cases, the explanation for this relatively high figure could be that only the most susceptible individuals will develop disease when the probability of infection decreases to very low levels.

While the size of the leprosy problem in Zimbabwe has decreased, and will be reduced further when over 1000 lepromatous patients will be discharged from follow-up during the next two years, the costs of running the programme have remained unchanged. In view of the ever-decreasing cost-effectiveness, the integration of the programme into the general health services has thus become imperative.

\section{Impediments to decentralization of the leprosy control programme}

In the decentralization process leprosy has to compete with a great number of other health problems, many of which are acute and extensive, killing rather than disabling, and far less costly and complicated to treat or prevent. Securing dedication to the leprosy patient and his problems is no doubt further impeded by the fact that little attention has been directed in the past to stimulating interest in leprosy among medical and paramedical health personnel.

A second obstacle to integration may be the leprosy patient himself, who is used to travelling by bus warrant to a clinic where a special leprosy officer has come from the provincial capital especially to see him and other leprosy patients. In the decentralized programme he will be attended by 'only' a nurse or health assistant. Given, in addition, the fragile ego of the average leprosy patient, it may be expected that decentralization will cause an increase in the defaulter rate.

The gravest impediments, however, lie in the domain of personnel and of its training. There is still a great shortage of health personnel in the country, and especially of staff trained in leprosy. The in-service training of general health staff has been going at a much slower rate than was anticipated. The Programme has been conducting one-month training courses at the Leprosy Hospital in Harare once or twice a year. As a result, two or three nurses or health assistants able to deal with leprosy patients can now be found in almost all districts. They are carrying out the leprosy work within the setting of their regular duties. Undeniably, the employment of such 'special' staff for leprosy control maintains a degree of verticality. It is questionable, however, whether any further integration is a commendable objective; given the present incidence of leprosy, the employment of two to three workers per district would mean each diagnosing, on average, one or two new cases annually. It seems implausible that health workers can maintain the necessary skills for recognizing, treating and following-up leprosy patients when these skills are addressed at such low rates. 
An unfortunate sequel of the Programme's accomplishment in training a number of general health workers in almost every district is that many district medical officers, health inspectors and nursing officers cannot supervise and assess the leprosy work of their staff, as they were not trained in the subject themselves. This contributes to the situation being somewhat unstable. In addition, transfers, resignations and appointments to other duties of the leprosy staff regularly disrupt the continuity of the services. Instruction of the senior district health staff is now considered a priority of the training programme. However, most can ill-afford to leave the district for a training course at the Leprosy Hospital in Harare; besides, in view of the size of the problem in their district, many are little inclined to appreciate their training in leprosy as a matter of urgency. The best that can normally be achieved is a one-day or two-day workshop in the district, preferably including the local leprosy clinic day. As there are 55 districts in the country, this is a very time-consuming enterprise. Evidently, these training activities should be conducted especially in areas of high leprosy prevalence (see Figure 1).

The satisfactory integration of leprosy control into the general health services seems attainable only by transferring the theoretical and practical training in leprosy to the medical and paramedical training schools - a process that will take another 10 to 15 years. It has already been accomplished for the medical undergraduates, who have been receiving instruction in leprosy since 1984. For six mornings they are attached to the outpatient clinic of the Leprosy Hospital. Although not much, it seems sufficient to familiarize them with the problems of leprosy and leprosy control, and with the management of the most common complications of the disease, such as neuritis and foot

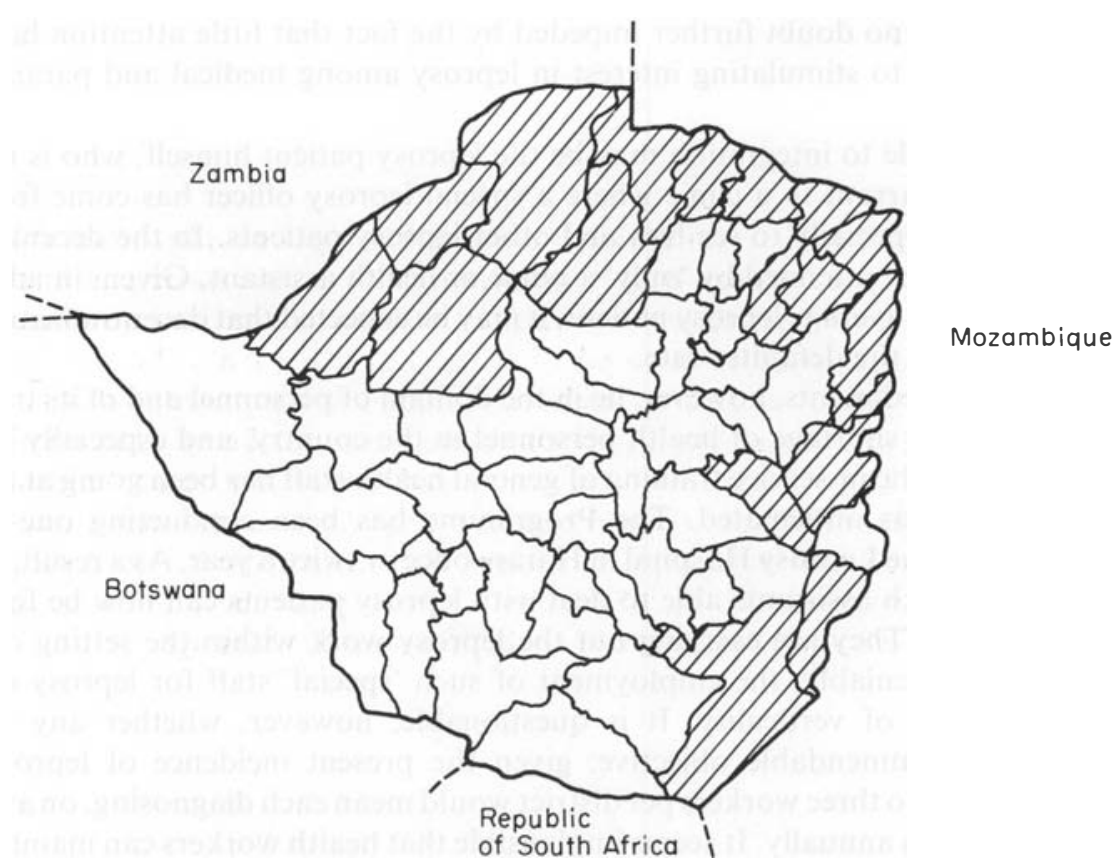

Figure 1. Zimbabwean administrative districts. Those districts in which the prevalence of leprosy is high are shaded. 
ulcers. Therefore since 1985 all Zimbabwean doctors that have graduated should be able to participate in the care of leprosy patients.

In the paramedical training schools the time allocated to leprosy in the curriculum has been, until now, usually left unused or allocated to other subjects in the absence of tutors trained and experienced in leprosy. Some PLOs have already been assisting at schools in their province. In view of the declining patient load in all provinces, participation in the instruction of trainee general health staff has now been identified as the first priority of the PLOs. As they have ready access to patients, their tutoring offers the great advantage of permitting students to become involved in the practical examination and health education of the patients, the taking of skin smears, and in the execution of administrative aspects, e.g. registers, treatment cards.

However, the decline of the patient load is also beginning to show in the field of teaching, as fewer and fewer patients are becoming available for practical demonstrations, especially with respect to the early signs of the disease, and of complications such as reversal reaction and ENL. Photographic material cannot, on the whole, replace the living patient, and is of very restricted value if the student has not become familiar with the manifestations of leprosy at first hand. Consequently, it seems that it will gradually become more and more difficult for trainee health workers in Zimbabwe to acquire sufficient knowledge and skills for the early diagnosis of leprosy, of early stages of neuritis with loss of nerve function, and of other complications. That may well have its repercussions on the quality of future leprosy control in the country. The scope of this problem and its trend could be assessed by the careful monitoring of the disability proportion among the new cases registered during the following years. Therefore it will be vital to ensure that registration and reporting in the decentralized programme should continue to be reliable.

\section{Conclusion}

Clearly Zimbabwe finds itself in an enviable position having such a low rate of leprosy incidence. It appears to have been largely unforeseeable that the successful implementation of the MDT regimen would provoke the hindrances to decentralization discussed in this paper. It is open to speculation whether the transition from the vertical to an integrated leprosy control programme might have been less complicated if it had been given first priority at the outset. As it happened, the initial intake of large numbers of patients induced not only a great workload but tended to generate an attitude amongst leprosy control staff that left little room for efforts directed towards integration.

It seems indubitable that most of the problems that leprosy control in Zimbabwe is now facing would have been avoided if training in leprosy had been introduced in the curricula of the medical and paramedical training schools 20 years ago. 